Geomatics, Natural Hazards and Risk

\title{
Observed Ionospheric Effects of 23 October 2011 Van, Turkey Earthquake
}

\author{
Feza Arikan , M. N. Deviren , O. Lenk, U. Sezen \& O. Arikan
}

To cite this article: Feza Arikan , M. N. Deviren , O. Lenk, U. Sezen \& O. Arikan (2012) Observed lonospheric Effects of 23 October 2011 Van, Turkey Earthquake, Geomatics, Natural Hazards and Risk, 3:1, 1-8, DOI: 10.1080/19475705.2011.638027

To link to this article: http://dx.doi.org/10.1080/19475705.2011.638027

\section{(2) Copyright Taylor and Francis Group, LLC}

曲 Published online: 17 Jan 2012.

Submit your article to this journal $\pi$

\section{Џll Article views: 146}

Q View related articles $ऍ$

4 Citing articles: 9 View citing articles 


\title{
Express Letter \\ Observed Ionospheric Effects of 23 October 2011 Van, Turkey Earthquake
}

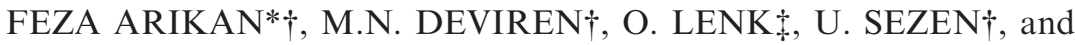 \\ O. ARIKAN $\S$
}

$†$ Department of Electrical and Electronics Engineering, Hacettepe University, Beytepe, 06800 Ankara, Turkey

\$General Command of Mapping, Defense Ministry, Cebeci, Ankara, Turkey $\S$ Department of Electrical and Electronics Engineering, Bilkent University, Bilkent, 06800 Ankara, Turkey

(Received 27 October 2011; in final form 1 November 2011)

\begin{abstract}
On 23 October 2011, a very strong earthquake with a magnitude of $\mathrm{Mw}=7.2$ shook Eastern Anatolia, and tremors were felt up to $500 \mathrm{~km}$ from the epicentre. In this study, we present an early analysis of ionospheric disturbance due to this earthquake using Global Positioning Satellite-Total Electron Content (GPSTEC). The variability with respect to average quiet day TEC (AQDT) and variability between the consecutive days are measured with symmetric KullbackLeibler divergence (SKLD). A significant variability in total electron content (TEC) is observed from the GPS stations in the $150 \mathrm{~km}$ neighbourhood of the epicentre eight and nine days prior to the earthquake. An ionospheric disturbance is observed from GPS stations even more than $1,000 \mathrm{~km}$ to the epicentre, especially those on the North Anatolian fault (NAF). The present results support the existence of lithosphere-atmosphere-ionosphere coupling (LAIC) associated with Van, Turkey earthquake.
\end{abstract}

\section{Introduction}

In recent years, increased earth and space-based observations of the ionosphere indicate that there exists a coupling mechanism between seismic activities in the lithosphere and deviations or disturbances in the electron concentrations in the ionosphere, especially before strong earthquakes. These observations usually include variability in the critical frequency of the F2 layer, foF2 and Total Electron Content (TEC) (Ondoh 2000, Chuo et al. 2001, Pulinets 2004, Karatay 2010). With a worldwide dense network, global positioning satellite (GPS) receivers offer a cost-effective and efficient way of computing TEC compared to expensive and sparse foF2 measurements from earth or space-based ionosondes (Arikan et al. 2003, Nayir et al. 2007). TEC is defined as the line integral of electron density on a path joining the satellite and the receiver (Arikan et al. 2003). The unit of TEC is given in TECU where $1 \mathrm{TECU}=10^{16} \mathrm{el} / \mathrm{m}^{2}$. In statistics and information theory, symmetric Kullback-Leibler divergence (SKLD) is a widely used measure of distance between

\footnotetext{
*Corresponding author. Email: arikan@hacettepe.edu.tr
} 
two probability density distributions (Cover and Thomas 2006). Previously, SKLD is applied to measure the difference between the experimental probability density functions (e-pdf) of average quiet day TEC (AQDT) and days prior to the earthquake, and it has been observed that GPS stations within $150-350 \mathrm{~km}$ to the epicentre demonstrate a significant variability nine to two days prior to the earthquake (Arikan et al. 2009, Karatay et al. 2009, Karatay 2010, Karatay et al. 2010a). According to the results of these studies, SKLD proved itself to be a better measure of disturbance or difference compared to cross correlation coefficient and L2-norm methods. Further, SKLD has been applied to earthquakes in North Anatolian Fault (NAF) using GPS recordings of Turkish National Permanent GPS Network (TNPGN) (Karatay 2010, Karatay et al. 2010b, 2010c, Özilhan 2010). Even with magnitude 4 or 5 earthquakes, close or on NAF, significant variability in TEC is observed using SKLD in comparisons with AQDT and consecutive days. In this study, the variability of Global Positioning Satellite-Total Electron Content (GPSTEC) prior to 23 October 2011 earthquake in Van is investigated using SKLD in comparisons with AQDT and consecutive days.

\section{Application of SKLD to detect TEC variability}

In this section, we will summarize the SKLD measurement method (Karatay 2010, Karatay et al. 2010a, Özilhan 2010). Let

$$
\mathbf{X}_{u ; d}=\left[\mathbf{X}_{u ; d}(1) \ldots . . \mathbf{X}_{u ; d}(n) \ldots . . \mathbf{X}_{u ; d}(N)\right]^{T}
$$

represent the set of TEC data of length $N$ estimated for day $d$ and GPS station $u$. Here, $n$ is the index where $1 \leq n \leq N$ and subscript $T$ is the transpose operator. In order to compare TEC values obtained from different seasons and days, data vectors as in equation (1) are normalized. The experimental probability density function (e-pdf) of TEC for station $u$ and day $d$ can be approximated using the TEC estimates as:

$$
\hat{\mathbf{P}}_{u ; d}=\mathbf{X}_{u ; d}\left[\sum_{n=N_{i}}^{N_{x}} x_{u ; d}(n)\right]^{-1}
$$

where $N_{i}$ and $N_{s}$ denote the initial and final indices for the measurement set, respectively. In order to compare the behaviour of TEC for the quiet days with those from the EQD, an AQDT estimate for each GPS station is obtained. For $N_{d}$ quiet days for station $u$, AQDT is defined as:

$$
\mathrm{X}_{u ; d_{\mathrm{i}}-d_{x}}=\frac{1}{N_{d}} \sum_{n_{d}=d_{i}}^{d_{x}} \mathrm{X}_{u ; n_{d}}
$$

where $n_{d}$ is the index for quiet day period (QDP) which extends from $d_{i}$ to $d_{s}$. Approximation for the e-pdf of AQDT is defined as follows:

$$
\hat{\mathbf{P}}_{u ; d_{i}-d_{x}}=\mathbf{X}_{u ; d_{i}-d_{x}}\left[\sum_{n=N_{i}}^{N_{x}} x_{u ; d_{i}-d_{x}}(n)\right]^{-1}
$$


The Kullback-Leibler (KL) divergences of normalized e-pdfs defined in equation (2) for day $d$ between stations $u$ and $v$ can be defined as:

$$
\begin{aligned}
& K L\left(\hat{\mathbf{P}}_{u ; d} \backslash \hat{\mathbf{P}}_{v ; d}\right)=\sum_{n=N_{i}}^{N_{x}} \hat{P}_{u ; d}(n) \ln \left(\frac{\hat{P}_{u ; d}(n)}{\hat{P}_{v ; d}(n)}\right) \\
& K L\left(\hat{\mathbf{P}}_{v ; d} \backslash \hat{\mathbf{P}}_{u ; d}\right)=\sum_{n=N_{i}}^{N_{x}} \hat{P}_{u ; d}(n) \ln \left(\frac{\hat{P}_{v ; d}(n)}{\hat{P}_{u ; d}(n)}\right)
\end{aligned}
$$

where $N_{i}<n<N_{s}$. The SKLD is defined as the sum of the KL divergences (Cover and Thomas 2006, Karatay 2010) as:

$$
K L D\left(\hat{\mathbf{P}}_{u ; d} ; \hat{\mathbf{P}}_{v ; d}\right)=K L\left(\hat{\mathbf{P}}_{u ; d} \backslash \hat{\mathbf{P}}_{v ; d}\right)+K L\left(\hat{\mathrm{P}}_{v ; d} \backslash \hat{\mathbf{P}}_{u ; d}\right)
$$

Using normalized AQDT, for day $d$ of station $u$, SKLD is defined as $K L \bar{D}\left(\hat{\mathrm{P}}_{u ; d}\right.$; $\left.\hat{\mathrm{P}}_{u ; d_{i}-d_{x}}\right)$. For consecutive days of station $u$, SKLD is defined as $K L D\left(\hat{\mathrm{P}}_{u ; d} ; \hat{\mathrm{P}}_{u ; d+1}\right)$.

\section{A summary of the 23 October 2011, Van Earthquake}

According to Kandilli Observatory and Earthquake Research Institute (KOERI) of Bogazici University, National Earthquake Monitoring Center (NECM), an earthquake of magnitude $\mathrm{Mw}=7.2(\mathrm{ML}=6.6)$ took place on 23 October 2011 at 10:41 UT (Universal Time) in Eastern Turkey to the northeast of Lake Van approximately $30 \mathrm{~km}$ to the north of the city Van with a population of 380,000 . The earthquake epicentre is located at $\left(38.7578^{\circ} \mathrm{N}, 43.3602^{\circ} \mathrm{E}\right)$ with a depth of $5.0 \mathrm{~km}$. The earthquake was felt within a $500 \mathrm{~km}$ radius and along the Iran-Turkey border region. The main shock has been followed by an intensive aftershock activity published at http://www.koeri.boun.edu.tr/scripts/lasteq.asp. The size of the largest aftershock recorded until present is $\mathrm{ML}=5.7$ on 23 October 2011 at 20:45 UT. The number of aftershocks above magnitude 3 has reached 307 as of 13:45 UT, 24 October 2011. Historically, Eastern Anatolia has suffered from severe earthquakes. The most recent one in the area occurred on 24 November 1976 at 12:22 UT, with $\mathrm{Ms}=7.5$ in Caldiran $\left(39.05^{\circ} \mathrm{N}, 44.04^{\circ} \mathrm{E}\right)$, close to Van. In figure 1 , the time series evolution of mura, a TNPGN station $43 \mathrm{~km}$ to the epicentre, is presented. The coseismic displacement due to the first shock is highly evident in north-south and east-west directions. In the following section, SKLD will be applied to TNPGN stations for 23 October 2011, Van earthquake.

\section{Results}

The possible seismic disturbances in the ionosphere due to the Van earthquake is investigated using the SKLD measure described in section 2. The Receiver INdependent EXchange (RINEX) data from GPS stations are obtained from the TNPGN. TNPGN is the reference station network of 146 continuously operating 


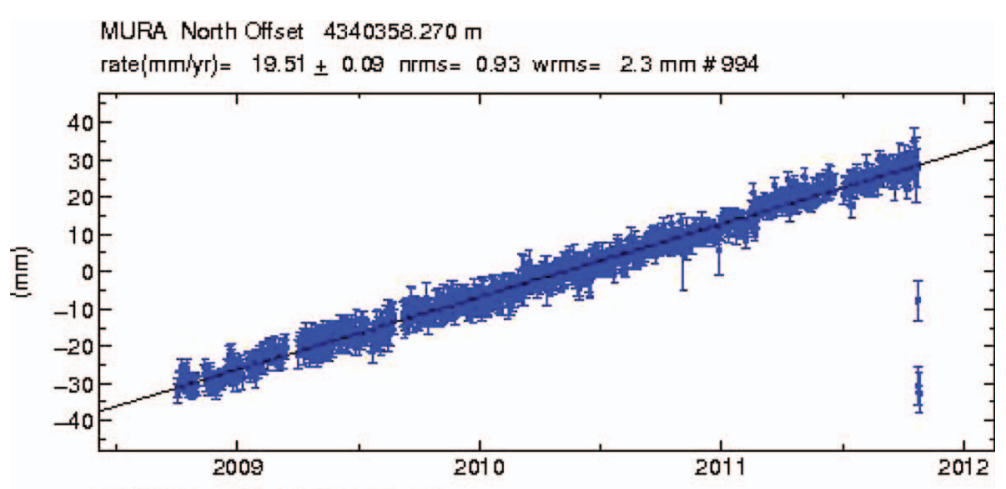

MURA East OHset $3786571.040 \mathrm{~m}$

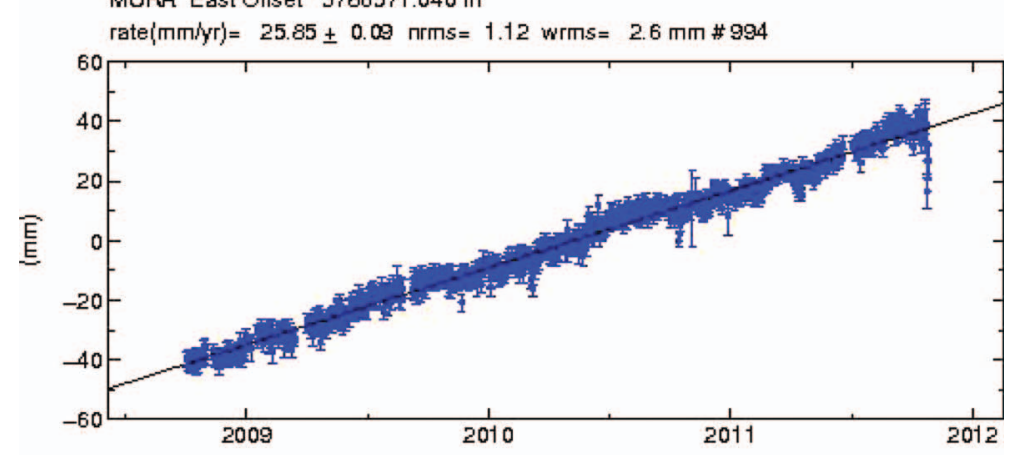

MURA Up Offset $1736.400 \mathrm{~m}$ rate $(\mathrm{mm} / \mathrm{lr})=7.69 \pm 0.35 \mathrm{nrms}=0.94 \mathrm{wrms}=8.5 \mathrm{~mm} \# 994$

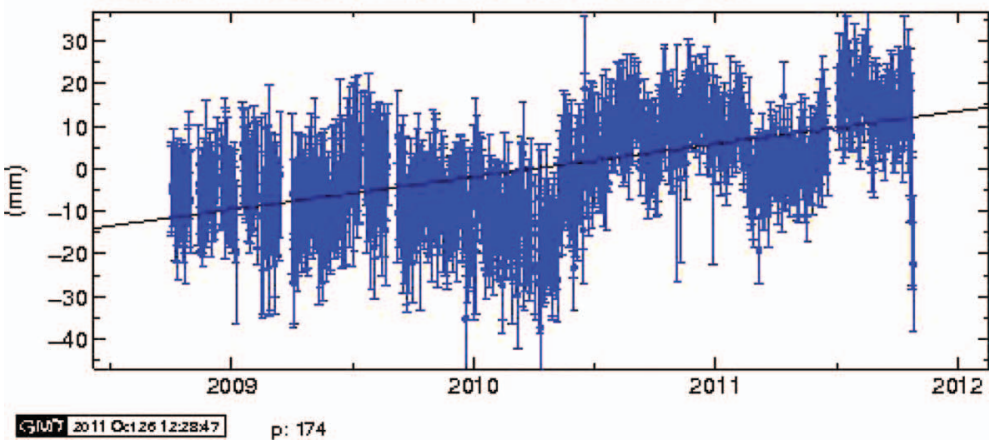

Figure 1. Survey mode observations and coseismic displacement due to the first shock for mura, $43 \mathrm{~km}$ to the epicentre.

GNSS stations (CORS) which are distributed uniformly across Turkey and North Cyprus Turkish Republic since May 2009. The GPS-TEC values for each station are estimated by IONOLAB-TEC using the Reg-Est algorithm described in Arikan et al. (2003) and Nayir et al. (2007), www.ionolab.org with a time resolution of $2.5 \mathrm{~min}$. The missing values of TEC or SKLD in figures of this section are due to the lack of RINEX data for those stations and/or days.

AQDT is obtained using the IONOLAB-TEC in equation (4) from 25 to $28 \mathrm{March}$ 2011. AQDT is compared with a magnetically QDP of 25-28 April 2011 and also with EQD, 14-23 October 2011. Quiet days are chosen according to the Kp and Dst indices provided in http://wdc.kugi.kyoto-u.ac.jp, and also there are no recorded 
earthquakes during those periods in Turkey. The geomagnetic disturbance indices of $\mathrm{Kp}$ and Dst indicate that the EQD period is also magnetically quiet. Therefore, we expect that the disturbances in the ionosphere to be due to seismic activities.

The 146 GPS stations in TNPGN are divided into five categories with respect to the distance to the epicentre. The first zone, Z1, includes stations which are within $150 \mathrm{~km}$ radius of the epicentre; $Z 2$ includes stations which are $150-360 \mathrm{~km}$ to the epicentre; Z3 stations are within $360-550 \mathrm{~km}$ to the epicentre; Z4 zone includes stations within $550-780 \mathrm{~km}$ to the epicentre and finally, Z5 have stations whose distances to the epicentre is larger than $780 \mathrm{~km}$. An AQDT is obtained for each of these stations and it is compared with QDP and EQD using SKLD. It is observed that for all the stations in the network, SKLD of EQD $(\mathrm{Se})$ is significantly larger than SKLD of QDP $(S q)$, on 14 and 15 October 2011, eight and nine days prior to the earthquake. The difference $\left(D_{u}=S e_{u}-S q_{u}\right)$ for each station is computed and then an average is taken within the zone, $D_{m}$. In table $1, D_{m}$ is presented for five zones for both 14 and 15 October 2011. It is observed that eight and nine days before the earthquake with 7.2 magnitude, $D_{u}=S e_{u}-S q_{u}$ values for all the stations in TNPGN network indicated a significant difference compared to the quiet days. The mean difference $D_{m}$ is highest in the first two zones that are close to the epicentre. $D_{m}$ reduces as the distance from the epicentre gets larger in zones Z3, Z4 and Z5. A comparison of SKLD of AQDT with QDP and EQD is also provided for four stations in figure 2. In figure $2, S e_{u}$ and mean $S q_{u}$ values of mura, surf, klis and yenc are provided. The mura station is $43 \mathrm{~km}$, surf is $435 \mathrm{~km}$, klis is $596 \mathrm{~km}$ and yenc is $1,394 \mathrm{~km}$ from the epicentre. The yenc station is located on the western edge of NAF. The difference $D_{u}=S e_{u}-S q_{u}$ is significant for all stations either $43 \mathrm{~km}$ or $1,394 \mathrm{~km}$ from the epicentre. In figure 3, IONOLAB-TEC values are presented for mura for 23 October 2011 (earthquake day, dotted line), 15 October 2011 (eight days prior to the earthquake, solid line), 26 April 2011 (quiet day, dashed line) and AQDT (dash dot line). The significant increase in ionization levels is apparent starting from nine days prior to the earthquake, compared to AQDT and quiet day TEC. Eight days prior to the earthquake, TEC has the same level for the night hours and there is an increase in peak TEC for the day hours. Yet, on earthquake day, the ionization is very high even during night hours. Also, the uncharacteristic increase in figure 3 for earthquake day between 16:00 UT and 22:00 UT might be due to other effects. SKLD is also applied to find the difference of TEC between consecutive days, $K L D\left(\hat{\mathrm{P}}_{u ; d} ; \hat{\mathrm{P}}_{u ; d+1}\right)$. For each station starting with nine days prior to the earthquake, SKLD of consecutive days $K e_{u}$ are computed. Similarly, SKLD of consecutive days are computed for days in QDP as $K q_{u}$. In figure 4, $K e_{u}$ and mean of $K q_{u}$ are presented for four stations, namely mura, surf, klis and yenc. The SKLD are indicated for the first day for a day 1 and day 2 comparison. For example, the

Table 1. Dm, mean of the differences $D=S e-S q$ within a distance zone.

\begin{tabular}{lcc}
\hline Zone & $D_{m}$ for 14 October 2011 & $D_{m}$ for 15 October 2011 \\
\hline Z1 & 0.0452 & 0.0461 \\
Z2 & 0.0333 & 0.0517 \\
Z3 & 0.0245 & 0.0439 \\
Z4 & 0.0247 & 0.0351 \\
Z5 & 0.0233 & 0.0205 \\
\hline
\end{tabular}



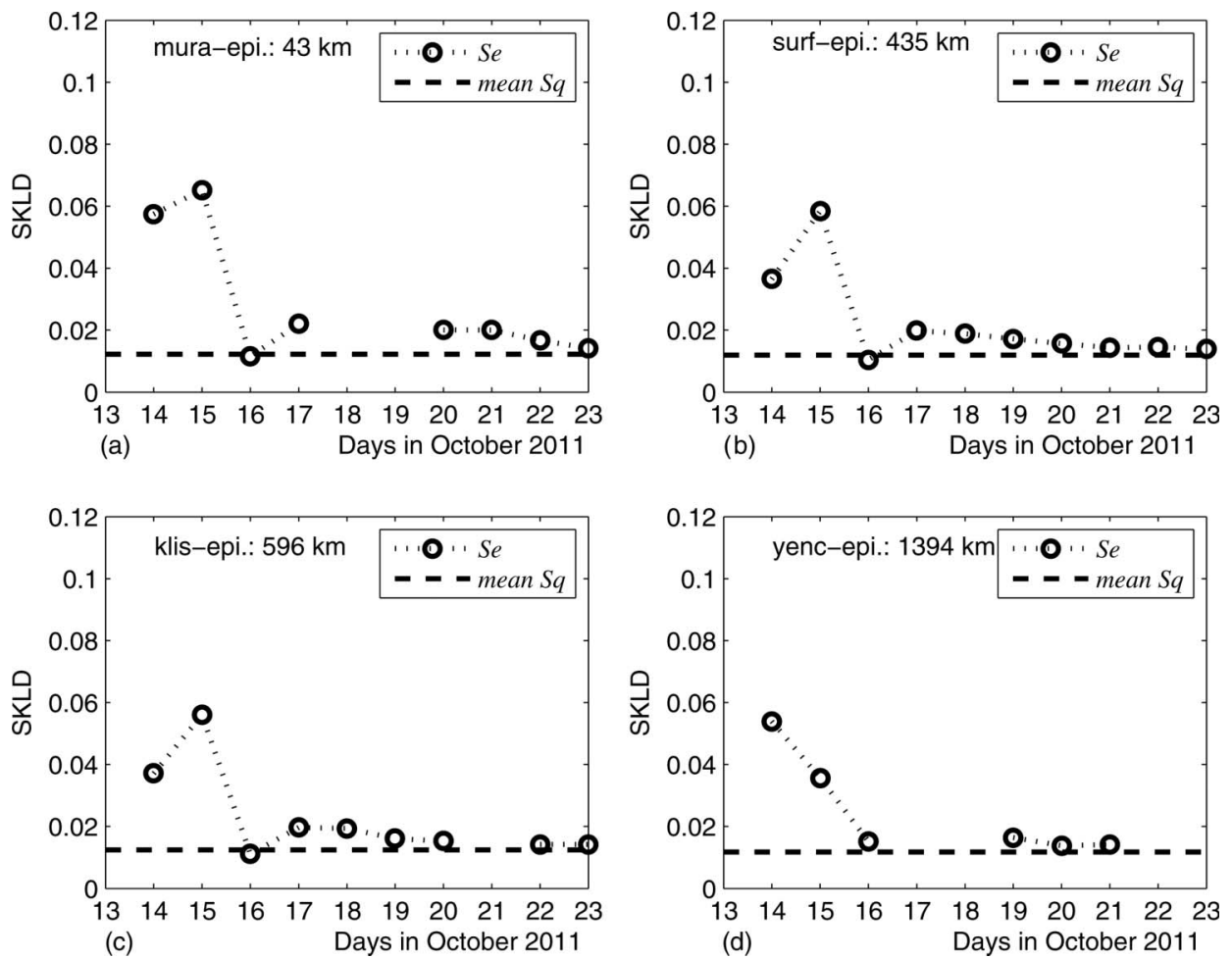

Figure 2. Comparison of SKLD values of $S e$ (AQDT with EQD) and mean of $S q$ (AQDT with QDP) (a) mura, (b) surf, (c) klis and (d) yenc.

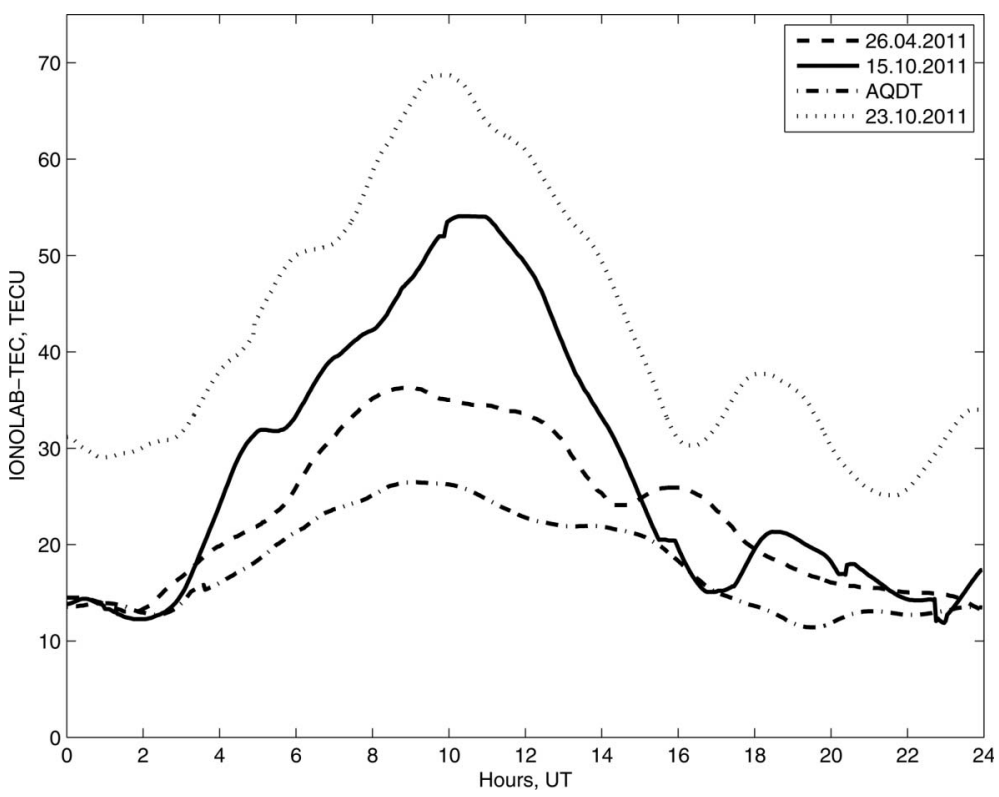

Figure 3. IONOLAB-TEC values for mura on 23 October 2011 (earthquake day, dotted line), 15 October 2011 (eight days prior to the earthquake, solid line), 26 April 2011 (quiet day, dashed line) and AQDT (dash dot line). 

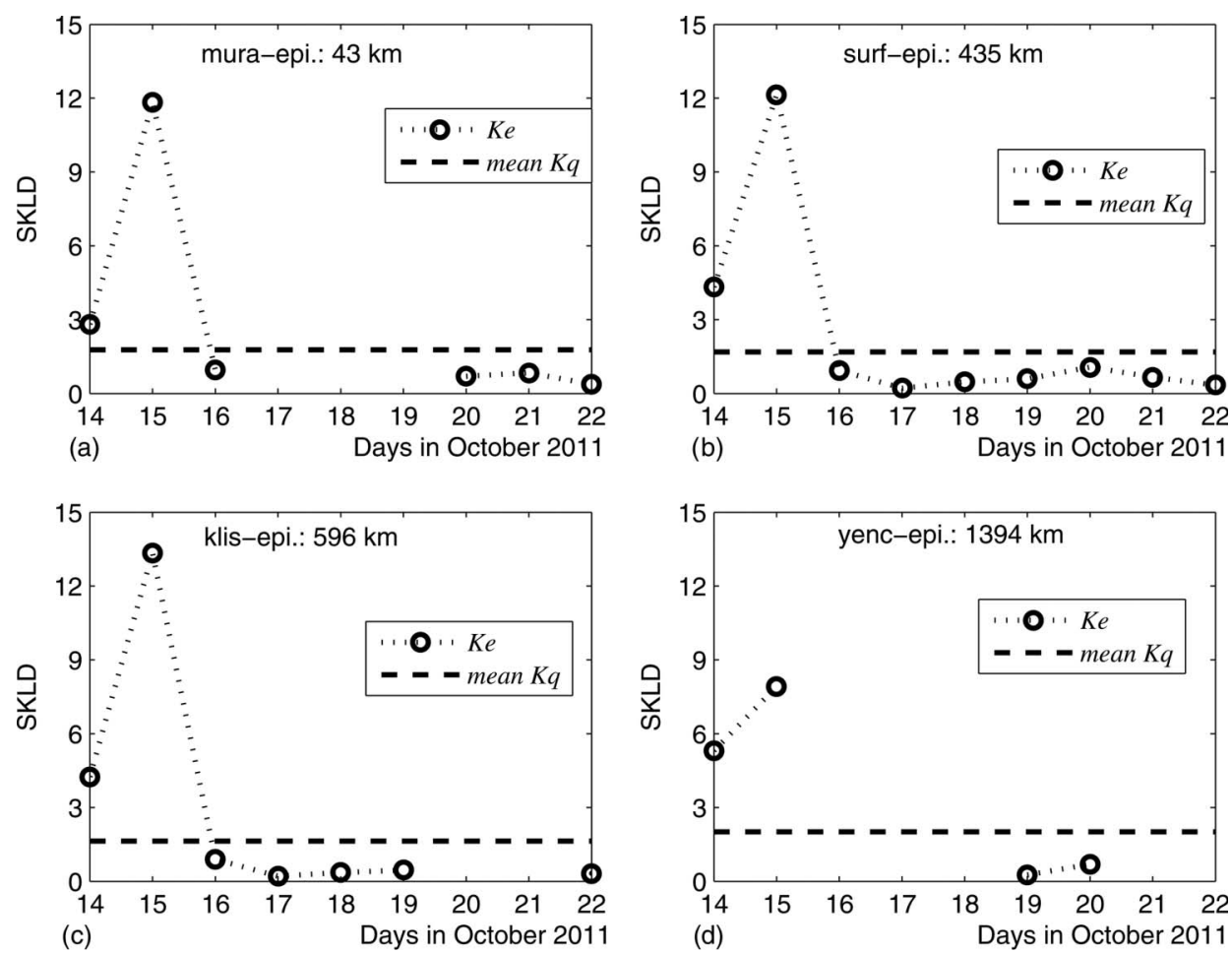

Figure 4. Comparison of SKLD values of consecutive days: $K e$ (EQD) and mean of $K q(\mathrm{QDP})$ for (a) mura, (b) surf, (c) klis and (d) yenc. The SKLD are indicated for the first day for a day 1 and day 2 comparison. For example, the comparison of 14 and 15 October is plotted for 14 October.

comparison of 14 and 15 October is plotted for 14 October and the comparison of 22 and 23 October is plotted for 22 October. It is observed that there is a major difference in consecutive EQD nine and eight days prior to the earthquake.

\section{Conclusions}

In this study, initial results for a possible the coupling of seismic activity to the ionosphere are presented for 7.2 magnitude earthquake in Van, Turkey that occurred on 23 October 2011. The variability of GPS-TEC between EQD and quiet days, and also between consecutive days prior to the earthquake is investigated using SKLD. In previous studies, for comparison between AQDT and EQD for earthquakes with magnitudes 4 and 5 on NAF, it is observed that SKLD is a strong candidate for developing an earthquake precursor tool for the stations that are located less than $150 \mathrm{~km}$ from the earthquake zones. In Van earthquake with magnitude 7.2, even stations $1,394 \mathrm{~km}$ from the epicentre on the NAF deviated significantly from the quiet day threshold. In the comparison of the consecutive days for each station, similar results are obtained. These initial results demonstrate that SKLD can be developed into a precursor tool for distinguishing seismic activity with a long-term constant analysis. Detailed geodetic analysis of displacement of GPS stations in TNPGN for Van earthquake is prepared by General Command of Mapping. For 
future studies, the earthquake precursor signal has to be identified as residuals in a local area over a known fault zone with a dense GPS receiver grid. Observation space, probabilistic transition mechanism and the thresholds will be formed with constant monitoring.

\section{Acknowledgements}

This study is supported by TUBITAK EEEAG grant no. 109E055. The authors wish to thank the anonymous referee for his/her careful revision of the manuscript.

\section{References}

Arikan, F., Erol, C.B. and Arikan, O., 2003, Regularized estimation of vertical total electron content from global positioning system data. Journal of Geophysical Research-Space Physics, 108, pp. 1469-1480.

Arikan, F., Karatay, S. and Arikan, O., 2009, Investigation of ionospheric disturbance due to strong earthquakes using total electron content. In Geophysical research abstracts, Vol. 11, EGU2009-8440, EGU General Assembly, 19-24 April 2009, Vienna, Austria.

Chuo, Y.J., Chen, Y.I., Liu, J.Y. and Pulinets, S.A., 2001, Ionospheric foF2 variations prior to strong earthquakes in Taiwan area. Advances in Space Research, 27, pp. 1305-1310.

Cover, T.M. and Thomas, A.J., 2006, Elements of Information Theory (New York, NY: Wiley Interscience Publishers).

Karatay, S., Arikan, F. and Arikan, O., 2009, Investigation of hourly and daily patterns for lithosphere-ionosphere coupling before strong earthquakes. In Proceedings of the Recent Advances in Space Technologies, RAST-2009, 11-13 June 2009, Istanbul, Turkey.

KARATAY, S., 2010, Investigation of relationship between earthquakes and ionospheric total electron content (in Turkish). PhD thesis, Firat University, Elazig, Turkey.

Karatay, S., Arikan, F. and Arikan, O., 2010a, Investigation of total electron content variability due to seismic and geomagnetic disturbances in the ionosphere. Radio Science, 45, p. RS5012.

Karatay, S., Arikan, F. and Arikan, O., 2010c, Comparison of ionospheric variations in seismic, geomagnetically disturbed and quiet days by IONOLAB-TEC estimates. In Abstracts of the Workshop on Electromagnetic Signals Associated with Earthquakes and Volcanoes, EMSEV 2010, 3-6 October 2010, Chapman University, Orange, CA, USA, pp. 92.

Karatay, S., Arikan, F., Arikan, O., Lenk, O., Aktug, B. and Aysezen, M.S., 2010b, Comparison of ionospheric variations in seismic and quiet days by CORS-TR IONOLAB-TEC estimates. In Proceedings of The International Beacon Satellite Symposium, BSS2010, 7-11 June 2010, Campus Nord UPC, Barcelona, Spain.

Nayir, H., Arikan, F., Arikan, O. and Erol, C.B., 2007, Total electron content estimation with Reg-Est. Journal of Geophysical Research-Space Physics, 122, p. A11313.

Ondoh, T., 2000, Seismo-ionospheric phenomena. Advances in Space Research, 26, pp. 12671272.

ÖZILHAN, B.E., 2010, Investigation of possible relationships between North Anatolian Fault zone earthquakes and ionosphere total electron content estimates (in Turkish). MS thesis, Hacettepe University, Ankara, Turkey.

Pulinets, S.A., 2004, Ionospheric precursors of earthquakes - Recent advances in theory and practical applications. TAO, 15, pp. 413-435. 